\title{
ÉDITORIAL
}

MARCEL JOLLIVET, JEAN-MARIE LEGAY et GÉrARD MÉGIE

\section{Sciences du vivant et Société : questions actuelles}

V

oici que l'actualité met à nouveau pleins feux, et de diverses manières, sur les problèmes que soulèvent les rapports entre les sciences du vivant et les sociétés. Le parlement français est en train de mettre fin à trois années de valse-hésitation, signe s'il en est d'une réelle perplexité politique, en votant une série de textes constituant une nouvelle législation sur la bioéthique. $\mathrm{Ce}$ vote va clore, pour un temps au moins, les débats sur des questions essentielles, qui ont connu ces derniers mois une vivacité particulière (encore que limitée à des cercles très restreints). Natures - Sciences - Sociétés reviendra sur le bilan qu'il faut tirer de cet épisode dans le travail constant d'ajustement qui doit se faire entre l'évolution des connaissances dans la biologie de la reproduction et l'évolution des sociétés.

Toujours en France, on pensait l' " affaire du sang contaminé » close par les décisions de justice. Les pétitions demandant la grâce des condamnés et les nouvelles plaintes pour " empoisonnement » déposées contre trois anciens ministres rouvrent le dossier. Au départ franco-françaises, les controverses lancées par les pétitions ont très rapidement pris une ampleur internationale, avec l'intervention de prix Nobel d'un côté et de mille cinq cents chercheurs de tous pays. Cette mobilisation rapide est l'expression de tout un tissu de relations contradictoires dans les domaines tant scientifique que socio-économique ; prenant les allures de ce qu'il faut bien considérer comme une forme de protestation d'une partie de la communauté scientifique, elle traduit sans doute aussi un malaise de fond. La question est à nouveau posée des rapports entre l'état des connaissances scientifiques et la décision politique, avec toutes ses composantes, dans un contexte d'incertitudes sur tous les plans.

Mais une autre question se profile derrière la démarche choisie par les signataires; c'est celle du rapport entre les scientifiques, la société civile et l'État de droit. Les scientifiques sont tout à la fois des citoyens ordinaires (donc soumis au respect de la " chose jugée "), les membres de " corporations » (avec ce que ceci implique d'intérêts matériels et moraux communs et donc de propension à se constituer en groupes de pression) et des professionnels dotés d'une compétence particulière leur conférant des responsabilités particulières (et sollicités en tant que tels). Comment traiter socialement les contradictions qui ne peuvent manquer de découler de ce triple statut ? | apparaît urgent de clarifier les choses sur ces points, car la question a une portée générale et surtout journalière, c'est-à-dire bien au-delà des crises, qui seules atteignent le grand public. À terme, c'est l'ouverture des chercheurs - et donc de la recherche - sur la société qui est en jeu. Là aussi, Natures Sciences - Sociétés apportera sa contribution.

Pour terminer ce tour d'horizon, il faut signaler l'information selon laquelle des expérimentations utilisant des produits radioactifs auraient été réalisés sur l'homme aux États-Unis dans les années cinquante. Au-delà de l'émotion légitime que peut susciter une telle information - surtout si l'on se réfère à la période à laquelle elle renvoie -, c'est encore deux autres questions qui sont posées et non des moindres puisqu'il ne s'agit de rien de moins que de celle de la transparence de la recherche et donc du rapport entre la recherche et de la démocratie (il semple en effet que le secret le plus total ait entouré à l'époque les pratiques dénoncées aujourd'hui) et de celle de l'expérimentation sur l'homme. Au moment où l'expérimentation sur l'animal est ellemême remise en question, n'est-il pas surprenant d'avoir à se demander ce qu'il en est réellement de cette dernière? 